\title{
Some Remarks on the Location of Zeroes of the Partition Function for Lattice Systems
}

\author{
David Ruelle \\ I.H.E.S., Bures-sur-Yvette, France
}

Received February 15, 1973

\begin{abstract}
We use techniques which generalize the Lee-Yang circle theorem to investigate the distribution of zeroes of the partition function for various classes of classical lattice systems.
\end{abstract}

\section{Introduction}

The Lee-Yang circle theorem [12] remains one of the very few effective tools which are at our disposal in the rigorous theory of phase transitions. An important conceptual clarification of this theorem, as well as an extension to quantum systems were given by Asano [2]. This work was continued by Suzuki-Fisher [19]. A generalization of the LeeYang theorem to noncircular regions by the present author [17] also benefitted from Asano's ideas. Here some more facts concerning the position of zeroes of the partition function $Z$ for lattice systems are presented. In particular, results due to Heilmann-Lieb [11], Heilmann [10], and Runnels-Hubbard [18] are recovered. Although there is as yet no general method for locating the zeroes of $Z$, the techniques known so far permit to say something in a fairly large number of cases.

The grand partition function $Z$ for a lattice gas is a polynomial in one complex variable $z$ (the activity). To locate the zeroes of $Z(z)$ it is convenient, as Lee and Yang already remarked, to work with a polynomial $P$ in $n$ variables such that

$$
Z(z)=P(z, \ldots, z)
$$

and to prove that $P\left(z_{1}, \ldots, z_{n}\right) \neq 0$ when the $z_{i}$ are away from certain regions of the complex plane. $P\left(z_{1}, \ldots, z_{n}\right)$ is the grand partition function for a system having a different activity $z_{i}$ at each lattice site, it is a polynomial of first degree in each argument separately.

Let $\Lambda$ be a finite lattice subset and $P$ the partition function for $\Lambda$ with some given interaction between sites. The arguments of $P$ are the $z_{x}$ with $x \in \Lambda$ and

$$
P=\sum_{X \subset A} e^{-\beta U(X)} \prod_{x \in X} z_{x}
$$


where $U(X)$ is the energy corresponding to the occupation of the subset $X$ of $\Lambda$. Suppose that $\Lambda^{\prime}$ and $\Lambda^{\prime \prime}$ are two lattice subsets and $P^{\prime}, P^{\prime \prime}$ the corresponding partition functions with energy functions $U^{\prime}$ and $U^{\prime \prime}$. It will be convenient to denote by $z_{x}^{\prime}$ the arguments of $P^{\prime}$ and by $z_{x}^{\prime \prime}$ the arguments of $P^{\prime \prime}$. We consider the partition function $P$ for $\Lambda^{\prime} \cup \Lambda^{\prime \prime}$ with energy $U^{\prime}+U^{\prime \prime}$ :

$$
P=\sum_{X \subset A^{\prime} \cup A^{\prime \prime}} e^{-\beta U^{\prime}\left(X \cap A^{\prime}\right)-\beta U^{\prime \prime}\left(X \cap A^{\prime \prime}\right)} \prod_{x \in X} z_{x} .
$$

Asano remarked that $P$ can be obtained from $P^{\prime}$ and $P^{\prime \prime}$ by the following rules.

(a) Take the product $P^{\prime} P^{\prime \prime}$.

(b) If $x \notin \Lambda^{\prime} \cap \Lambda^{\prime \prime}$ replace $z_{x}^{\prime}$ or $z_{x}^{\prime \prime}$ in the product by $z_{x}$.

(c) If $x \in \Lambda^{\prime} \cap \Lambda^{\prime \prime}$ contract $z_{x}^{\prime}$ and $z_{x}^{\prime \prime}$ to $z_{x}$ : we start from a polynomial of first degree separately with respect to $z_{x}^{\prime}$ and $z_{x}^{\prime \prime}$, i.e., of the form

$$
a+b z_{x}^{\prime}+c z_{x}^{\prime \prime}+d z_{x}^{\prime} z_{x}^{\prime \prime}
$$

and contraction means replacing it by

$$
a+d z_{x} .
$$

As it turns out the position of the zeroes of a contracted polynomial is related to the position of the zeroes of the original polynomial, and therefore knowledge of the position of the zeroes of $P^{\prime}$ and $P^{\prime \prime}$ yields some information on the zeroes of $P$. In Section 1 we shall present the relevant theorems on polynomials. In Section 2 we shall consider some applications. In Section 3 we shall look in more details at the situation originally considered by Lee and Yang, where the zeroes of $Z(z)$ lie on the circle $|z|=1$.

\section{General Theorems}

The results in this section are mostly not new, they are collected here for convenience.

1.1. Theorem [17]. Let $\Lambda^{\prime}, \Lambda^{\prime \prime}$ be finite sets and $P^{\prime}, P^{\prime \prime}$ be polynomials with complex coefficients. The variables of $P^{\prime}$ are $z_{x}^{\prime}$, with $x \in \Lambda^{\prime}$, and

$$
P^{\prime}=\sum_{X \subset A^{\prime}} c_{X}^{\prime} \prod_{x \in X} z_{x}^{\prime} .
$$

It is assumed that there exist closed subsets $M_{x}^{\prime}$ of the complex plane such that $0 \notin M_{x}^{\prime}$ and $P^{\prime} \neq 0$ when

$$
z_{x}^{\prime} \notin M_{x}^{\prime} \text { for all } x \in \Lambda^{\prime} .
$$

Similar assumptions hold for $P^{\prime \prime}$. Define

$$
P=\sum_{X \subset A^{\prime} \cup A^{\prime \prime}} c_{X \cap A^{\prime}}^{\prime} \mathcal{C}_{X \cap A^{\prime \prime}}^{\prime \prime} \prod_{x \in X} z_{x} .
$$


Then $P \neq 0$ when ${ }^{1}$

$$
z_{x} \notin\left\{\begin{array}{lll}
M_{x}^{\prime} & \text { for } & x \in \Lambda^{\prime} \backslash \Lambda^{\prime \prime}, \\
M_{x}^{\prime \prime} & \text { for } & x \in \Lambda^{\prime \prime} \backslash \Lambda^{\prime}, \\
-M_{x}^{\prime} M_{x}^{\prime \prime} & \text { for } & x \in \Lambda^{\prime} \cap \Lambda^{\prime \prime} .
\end{array}\right.
$$

To prove this theorem, one obtains $P$ from $P^{\prime} P^{\prime \prime}$ by successive contractions, applying the following fact

1.2. Lemma [17]. Let $M^{\prime}, M^{\prime \prime}$ be closed subsets of the complex plane, not containing 0 . Suppose that the polynomial

$$
a+b z^{\prime}+c z^{\prime \prime}+d z^{\prime} z^{\prime \prime}
$$

can vanish only when $z^{\prime} \in M^{\prime}$ or $z^{\prime \prime} \in M^{\prime \prime}$, then

$$
a+d z
$$

can vanish only when $z \in-M^{\prime} M^{\prime \prime}$.

By successive applications of 1.1 one gets information about the zeroes of the polynomial $P_{A}$ for a "large" $A$ from similar knowledge about polynomials $P_{A_{1}}$ with "small" $\Lambda_{i}$. The following result yields a starting point.

1.3. Theorem $\left(\right.$ Grace $\left.^{2}\right)$. Let $Q(z)$ be a polynomial of degree $n$ with complex coefficients and $P\left(z_{1}, \ldots, z_{n}\right)$ the only polynomial which is symmetric in its arguments, of degree 1 in each, and such that

$$
P(z, \ldots, z)=Q(z) .
$$

If the roots of $Q$ are all contained in a closed circular region $M$, and $z_{1} \notin M, \ldots, z_{n} \notin M$, then $P\left(z_{1}, \ldots, z_{n}\right) \neq 0$.

A circular region is the inside or outside of a circle, or a half plane. We locate first the zeroes of $Q$ in a trivial case.

1.4. Lemma. If $a$ is real and $|a| \leqq 1$, then the zeroes of have modulus 1 .

$$
Q(z)=z^{2}+2 a z+1
$$

From this the Lee-Yang circle theorem follows:

1.5. Theorem (Lee-Yang $[12]^{3}$ ). Given real numbers $a_{x y}$, for $x, y \in \Lambda$ and $x \neq y$, such that $\left|a_{x y}\right| \leqq 1$,

(a) the polynomial

$$
P=\sum_{X \subset A}\left(\prod_{x \in X} z_{x}\right) \prod_{x \in X} \prod_{y \in \Lambda \backslash X} a_{x y}
$$

does not vanish when $\left|z_{x}\right|<1$ for all $x \in \Lambda$ or when $\left|z_{x}\right|>1$ for all $x \in \Lambda$.

\footnotetext{
${ }^{1}$ Notation: $-M^{\prime} M^{\prime \prime}=\left\{-z^{\prime} z^{\prime \prime}: z^{\prime} \in M^{\prime}\right.$ and $\left.z^{\prime \prime} \in M^{\prime \prime}\right\}$.

2 See Polya u. Szegö [16] V, Exercise 145.

3 The conclusions of this theorem hold under more general assumptions, see $[2,19]$, and Section 3 below.
} 
(b) the polynomial

$$
Z(z)=\sum_{X \subset A} z^{|X|} \prod_{x \in X} \prod_{y \in \Lambda \backslash X} a_{x y}
$$

where $|X|$ is the number of points in $X$, has all its zeroes on the unit circle.

(a) is proved by applying Theorem 1.1 repeatedly to contract the product of all polynomials

$$
P_{\{x, y\}}\left(z_{x}, z_{y}\right)=z_{x} z_{y}+a_{x y}\left(z_{x}+z_{y}\right)+1 .
$$

Because of 1.3,1.4, one can take every set $M$ to be the closed exterior of the unit circle. Therefore $P \neq 0$ when $\left|z_{x}\right|<1$ for all $x \in \Lambda$. When $\left|z_{x}\right|>1$ for all $x \in \Lambda$, then $\left|z_{x}^{-1}\right|<1$ and the symmetry of the polynomial gives again $P \neq 0$. (b) is an obvious consequence of (a).

We note a result similar to Theorem 1.3 for the polynomials $P$ satisfying the conclusions of the Lee-Yang circle theorem.

1.6. Proposition. Let $P\left(z_{1}, \ldots, z_{n}\right)$ be a complex polynomial which is different from 0 when $\left|z_{i}\right|<1$, all $i$, and when $\left|z_{i}\right|>1$, all $i$. Let

$$
Q(z)=P(z, \ldots, z) \text {. }
$$

If the roots of $Q$ are all contained in a closed circular region $M$, and $z_{1} \notin M, \ldots, z_{n} \notin M$, then $P\left(z_{1}, \ldots, z_{n}\right) \neq 0$.

This result is essentially contained in [13]. It can be proved by using the "double cone theorem" (see $[3,6]$ ) and then performing the analytic completion of the union of two polydiscs [9].

1.7. Example. Let $\sqrt{\sqrt{2}-1}<a<\sqrt{\sqrt{2}+1}$, then the polynomial

$$
\begin{aligned}
P\left(z_{1}, z_{2},\right. & \left.z_{3}, z_{4} ; a\right)=z_{1} z_{2} z_{3} z_{4} \\
& +a^{2}\left(z_{2} z_{3} z_{4}+z_{1} z_{3} z_{4}+z_{1} z_{2} z_{4}+z_{1} z_{2} z_{3}\right) \\
& +a^{2}\left(z_{1} z_{2}+z_{2} z_{3}+z_{3} z_{4}+z_{4} z_{1}\right)+a^{4}\left(z_{1} z_{3}+z_{2} z_{4}\right) \\
& +a^{2}\left(z_{1}+z_{2}+z_{3}+z_{4}\right)+1
\end{aligned}
$$

does not vanish when $\operatorname{Re} z_{1} \geqq 0, \operatorname{Re} z_{2} \geqq 0, \operatorname{Re} z_{3} \geqq 0, \operatorname{Re} z_{4} \geqq 0$.

If $a \leqq 1, P$ is of the type (1.1) and therefore, according to Proposition 1.6, it suffices to show that the roots of

$$
Q(z)=z^{4}+4 a^{2} z^{3}+\left(4 a^{2}+2 a^{4}\right) z^{2}+4 a^{2} z+1
$$

are contained in $\{z: \operatorname{Re} z<0\}$, but this is clear from the fact that

$$
Q(z)=\left[z^{2}+\left(2 a^{2}+\sqrt{2}\left(1-a^{2}\right)\right) z+1\right]\left[z^{2}+\left(2 a^{2}-\sqrt{2}\left(1-a^{2}\right)\right) z+1\right] .
$$

The case $a \geqq 1$ is handled by noticing that

$$
a^{-4} z_{2} z_{4} P\left(z_{1}, z_{2}^{-1}, z_{3}, z_{4}^{-1}\right)=P\left(z_{1}, z_{2}, z_{3}, z_{4} ; a^{-1}\right) .
$$


The following fact will prove useful.

1.8. Proposition. If the polynomials

and

$$
a_{n} z^{n}+\left(\begin{array}{c}
n \\
n-1
\end{array}\right) a_{n-1} z^{n-1}+\cdots+\left(\begin{array}{l}
n \\
1
\end{array}\right) a_{1} z+a_{0}
$$

$$
b_{n} z^{n}+\left(\begin{array}{c}
n \\
n-1
\end{array}\right) b_{n-1} z^{n-1}+\cdots+\left(\begin{array}{l}
n \\
1
\end{array}\right) b_{1} z+b_{0}
$$

have all their roots on the negative real axis, then the polynomial

$$
a_{n} b_{n} z^{n}+\left(\begin{array}{c}
n \\
n-1
\end{array}\right) a_{n-1} b_{n-1} z^{n-1}+\cdots+\left(\begin{array}{l}
n \\
1
\end{array}\right) a_{1} b_{1} z+a_{0} b_{0}
$$

has all its roots on the negative real axis.

This is a direct consequence of a theorem of Szegö ${ }^{4}$. We shall use it to locate the zeroes of a polynomial $Q$ of the type appearing in Theorem 1.3.

1.9. Proposition. If $\alpha>0$, all the roots of the polynomial

are real and negative. $\quad \sum_{k=0}^{n}\left(\begin{array}{l}n \\ k\end{array}\right) e^{-\frac{k(k-1)}{2} \alpha} z^{k}$

Clearly the polynomial

$$
\begin{aligned}
& \sum_{k=0}^{n}\left(\begin{array}{l}
n \\
k
\end{array}\right)\left(1-\frac{k(k-1)}{2} \alpha\right) z^{k} \\
= & (1+z)^{n}-\frac{n(n-1)}{2} \alpha z^{2}(1+z)^{n-2} \\
= & (1+z)^{n-2}\left[1+2 z+\left(1-\frac{n(n-1)}{2} \alpha\right) z^{2}\right] .
\end{aligned}
$$

has all its roots real and negative for small $\alpha>0$.

Using $N$ times Proposition 1.8 we find that

$$
\sum_{k=0}^{n}\left(\begin{array}{l}
n \\
k
\end{array}\right)\left(1-\frac{1}{N} \frac{k(k-1)}{2} \alpha\right)^{N} z^{k}
$$

has all its roots real and negative for small $\alpha / N>0$. It remains to let $N \rightarrow \infty$ to prove the proposition.

\subsection{Example. The polynomial}

$$
P\left(z_{1}, z_{2}, z_{3}, z_{4}\right)=1+z_{1}+z_{2}+z_{3}+z_{4}+z_{1} z_{3}+z_{2} z_{4}
$$

does not vanish when

$$
z_{1}, z_{2}, z_{3}, z_{4} \notin M=\left\{x+i y: y^{2} \geqq x^{2}+2 x+\frac{1}{2}\right\} .
$$

\footnotetext{
${ }^{4}$ See Polya and Szego [16], V, Exercise 151.
} 
This result is due to Runnels and Hubbard [18] and is obtained by noticing that $P \neq 0$ if

$$
\operatorname{Re}\left(\frac{1}{2}+z_{1}+z_{3}+z_{1} z_{3}\right)>0 \text { and } \operatorname{Re}\left(\frac{1}{2}+z_{2}+z_{4}+z_{2} z_{4}\right)>0 .
$$

The idea of this proof is quite different from the earlier considerations in this section, and might have other applications.

\section{Applications to Statistical Mechanics}

The use of the Lee-Yang circle theorem for ferromagnetic spin systems is well-known $[20,12]$, and the application of Theorem 1.1 to lattice gases has been described in $[17,18]$. We indicate here some further results.

2.1. Proposition. Let $2 J$ be the interaction energy between $a+$ and $a-$ spin at neighbouring sites in a 2-dimensional Ising model (interaction 0 for ++ and - - pairs). For the square lattice the free energy is an analytic function of the magnetic field $H$ when

$$
\frac{1}{2} \log (\sqrt{2}-1)<2 \beta J<\frac{1}{2} \log (\sqrt{2}+1) .
$$

For the triangular lattice the free energy is an analytic function of $H$ when

$$
0<2 \beta J<\frac{1}{2} \log \frac{3}{2}
$$

(notice that the exact critical temperature for $H=0$ is known to be given by $2 \beta|J|=\log (\sqrt{2}+1)$ for a square lattice and by $2 \beta J=\frac{1}{2} \log 3$ for $a$ triangular lattice).

Decomposing the square lattice into squares (see Fig.1), we can obtain the Ising partition function by contracting a product of polynomials of the form (1.2) (we write $a=e^{-2 \beta J}, z=e^{\beta H}$ ). The proposition follows then from Theorem 1.1 and Example 1.7. The triangular lattice is similarly decomposed into triangles (Fig. 2). In view of Theorems 1.3 and 1.5 , we

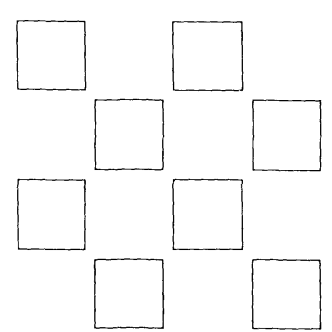

Fig. 1

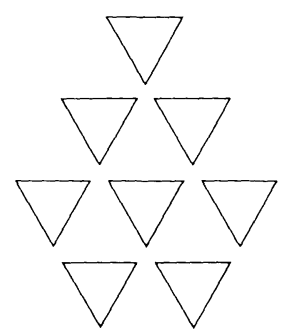

Fig. 2 
only have to show that the zeroes of

$$
Q(z)=z^{3}+3 z^{2} a^{2}+3 z a^{2}+1
$$

are not in the angular region

$$
|\arg z| \leqq \frac{2 \pi}{3} .
$$

This is checked immediately, since

$$
Q(z)=(z+1)\left(z^{2}+\left(3 a^{2}-1\right) z+1\right) .
$$

2.2. Theorem (Heilmann [10]). Let $Z$ be a line graph, i.e. $Z$ can be decomposed into finite subgraphs $Z_{i}$ and

(a) $Z_{i}$ consists of all line segments joining $n_{i}$ vertices

(b) each vertex of $Z$ belongs to only two different $Z_{i}$.

Consider a lattice gas with repulsive interactions between nearest neighbours on $Z$. We assume that the interaction energies are the same on the bounds of the same subgraph $Z_{i}$, they may otherwise be different. Under these conditions the thermodynamic functions are analytic in the activity $z$ for $z$ outside the negative real axis.

The following proof of Heilmann's theorem is based upon an idea of Runnels and Hubbard [18]. Let $P_{i}\left(z_{1}, \ldots, z_{n}\right)$ be the grand partition function for the subgraph $Z_{i}$. By Theorem 1.3 and Proposition 1.9, $P_{i}\left(z_{1}, \ldots, z_{n}\right) \neq 0$ when $z_{i} \notin M_{\theta}$ for all $i$, where

$$
M_{\theta}=\left\{z: \operatorname{Im} e^{i \theta}(z+c) \leqq 0\right\}
$$

for some $c>0$ and $-\frac{\pi}{2}<\theta<\frac{\pi}{2}$. Therefore, by Theorem 1.1, the grand partition function for a subgraph of $Z$ consisting of a finite union of subgraphs $Z_{i}$ has no zero when $\arg z_{x}=2 \theta$ for all vertices $x$. Taking all $z_{x}$ equal we find that the grand partition function vanishes only for $z$ real and negative.

2.3. Remarks. (a) The limit of nearest neighbour exclusion corresponds to the monomer-dimer problem treated by Heilmann and Lieb [11].

(b) If a line segment with attractive interaction is inserted between every pair of subgraphs $Z_{i}$ which had originally a vertex in common, the thermodynamic functions remain analytic in $z$ for $\operatorname{Re} z \geqq 0$. This is because the zeroes of the grand partition function of the attractive line segments have negative real part. This applies for instance to the graph of Fig. 3, where the heavy lines correspond to attractive interactions. 


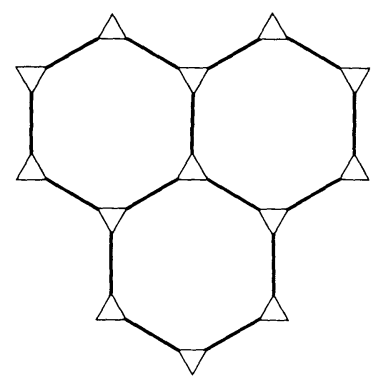

Fig. 3

2.4. Proposition ${ }^{5}$. Let $2 J$ be the interaction energy between $a+$ and a - spin at neighbouring sites in the 2-dimensional square Ising model (interaction 0 for ++ and -- pairs). If $H=0$, the free energy is an analytic function of $e^{-2 \beta J}$ for all complex $e^{-2 \beta J}$ such that $\left|e^{-2 \beta J}\right|$ $<(\sqrt{2}-1)^{2}$.

With appropriate boundary conditions (all spins + on the boundary), the partition function is a polynomial in $z=e^{-2 \beta J}$, the coefficient of $z^{n}$ being the number of closed polygons of length $n$ on the dual lattice. This polynomial can be obtained by contracting the product of polynomials $P_{x}$ corresponding to the vertices of the dual lattice. $P_{x}$ is a polynomial in the variables $z_{i}$ corresponding to the four bounds leading to $x$ :

$$
\begin{gathered}
P\left(z_{1}, z_{2}, z_{3}, z_{4}\right)=z_{1} z_{2} z_{3} z_{4}+z_{1} z_{2}+z_{2} z_{3}+z_{3} z_{4}+z_{4} z_{1} \\
+z_{1} z_{3}+z_{2} z_{4}+1 .
\end{gathered}
$$

By Theorem 1.3, $P_{x}$ is different from zero if $z_{1} \notin M, \ldots, z_{4} \notin M$, where $M$ is a closed circular region containing the roots of

$$
Q(z)=z^{4}+6 z^{2}+1,
$$

i.e. $\pm i(\sqrt{2} \pm 1)$. Therefore, by Theorem 1.1, the partition function is different from 0 when $|z|<(\sqrt{2}-1)^{2}$.

2.5. Remarks. (a) It has been conjectured that the zeroes of the partition function in the above proposition lie asymptotically on the two circles $|z \pm 1|=\sqrt{2}$. This conjecture (see Fisher [7]) is supported by numerical evidence (see [1]). Notice that 2.4 follows from Onsager's exact solution, but that extensions of the above proof to other lattices are possible.

5 The idea of this proposition is due to Miracle-Sole [14].

6 We may also notice that $2 P=\left(z_{1}+1\right)\left(z_{2}+1\right)\left(z_{3}+1\right)\left(z_{4}+1\right)+\left(z_{1}-1\right)\left(z_{2}-1\right)\left(z_{3}-1\right)$ $\cdot\left(z_{4}-1\right)$ and therefore $P \neq 0$ when $-\frac{\pi}{4}<\arg \left[\left(z_{1}-1\right) /\left(z_{1}+1\right)\right]<\frac{\pi}{4}$ for $i=1,2,3,4$. This yields a slight extension of the region $|z|<(\sqrt{2}-1)^{2}$. 
(b) Consider a 2-dimensional square Ising antiferromagnet. For large $\beta$ and sufficiently small $|H|$, there exist at least two different equilibrium states (see Dobrushin [5] and also [8, 4]). It is now also known that the grand partition function is free from zeroes in this region. (Brascamp and Kunz, private communication).

(c) More generally, one would like to know if the zeroes of the grand partition function tend to stay on lines which intersect the positive real axis at a few points, as suggested by Lee and Yang [20], or if they behave in other ways (filling 2-dimensional regions or clustering on the positive real axis). One dimensional systems with finite range forces can be handled (see for instance [15] and references quoted there) but are of limited interest since they do not exhibit phase transitions.

\section{Complements to the Lee-Yang Theorem}

The main purpose of this section is to show that the conclusions of the Lee-Yang circle theorem remain valid under small even (many-body) perturbations of the interaction. For a precise statement see Proposition 3.8. We start with some general results closely related to the work of Asano [2] and Suzuki-Fisher [19].

We shall use the notation

$$
\mathbb{D}=\{\zeta \in \mathbb{C}:|\zeta| \leqq 1\}, \quad \partial \mathbb{D}=\{\zeta \in \mathbb{C}:|\zeta|=1\} .
$$

Let $P$ be a complex polynomial in several variables which is of degree 1 with respect to each, i.e., $A$ is a finite set and

$$
P\left(z_{A}\right)=\sum_{X \subset A} c_{X} z^{X}
$$

where $z_{\Lambda}=\left(z_{x}\right)_{x \in A}, z^{X}=\prod_{x \in X} z_{x}$. We introduce the condition

(A) If $z_{A} \in \mathbb{D}^{|\Lambda|}$ and $P\left(z_{A}\right)=0$, then $z_{\Lambda} \in(\partial \mathbb{D})^{|\Lambda|}$.

Let now $P^{\dagger}$ be defined by $P^{\dagger}\left(z_{A}\right)=\sum_{X \subset A} c_{A \backslash X}^{*} z^{X}$ where * denotes complex conjugation. Notice that

$$
\left[P\left(z_{\Lambda}\right)\right]^{*}=\sum_{X \subset A} c_{\Lambda \backslash X}^{*} z^{* A \backslash X}=z^{* \Lambda} p^{\dagger}\left(z_{\Lambda}^{*-1}\right) .
$$

For any complex $\omega$ such that $|\omega|=1$, the mapping $P \rightarrow \omega P^{\dagger}$ is involutory: $\omega\left(\omega P^{\dagger}\right)^{\dagger}=P$. Having chosen $\omega$ we introduce the condition

(B) $P=\omega P^{\dagger}$.

In the applications to statistical mechanics, $\omega=1$ and the $c_{X}$ are real so that $(\mathrm{B})$ reduces to

$$
c_{X}=c_{A \backslash X} .
$$


3.1. Proposition. When $I \subset \Lambda$, define

$$
A^{(I)}\left(z_{I}\right)=\sum_{X \subset I} c_{X} z^{X}
$$

If $P$ satisfies $(\mathrm{A})$ and $I \neq \Lambda, A^{(I)}$ does not vanish on $\mathbb{D}^{|I|}$.

We have $A^{(I)}\left(z_{I}\right)=P\left(\tilde{z}_{A}\right)$ where $\tilde{z}_{x}=z_{x}$ for $x \in I$ and $\tilde{z}_{x}=0$ for $x \notin I$. When $z_{I} \in \mathbb{D}^{|I|}$ we have $\tilde{z}_{\Lambda} \in \mathbb{D}^{|\Lambda|}, \tilde{z}_{\Lambda} \notin(\partial \mathbb{D})^{|\Lambda|}$ and the lemma follows.

3.2. Proposition. Let $I, K,\{u\}$ be pairwise disjoint, and

Define

$$
\begin{aligned}
& R\left(z_{I \cup\{u\}}\right)=A\left(z_{I}\right)+B\left(z_{I}\right) z_{u} \\
& S\left(z_{K \cup\{u\}}\right)=C\left(z_{K}\right)+D\left(z_{K}\right) z_{u} .
\end{aligned}
$$

$$
P\left(z_{I \cup K \cup\{u\}}\right)=A\left(z_{I}\right) C\left(z_{K}\right)+B\left(z_{I}\right) D\left(z_{K}\right) z_{u} .
$$

If $R$ and $S$ satisfy (A), then $P$ satisfies (A).

Let $z_{I \cup K \cup\{u\}} \in \mathbb{D}^{|I|+|K|+1}$ and $P\left(z_{I \cup K \cup\{u\}}\right)=0$. If $R, S$ satisfy (A) we have

$$
\left|B\left(z_{I}\right) / A\left(z_{I}\right)\right| \leqq 1, \quad\left|D\left(z_{K}\right) / C\left(z_{K}\right)\right| \leqq 1
$$

with equality only if $z_{I} \in(\partial \mathbb{D})^{|I|}, z_{K} \in(\partial \mathbb{D})^{|K|}$. This is compatible with

$$
1 \leqq\left|z_{u}^{-1}\right|=\frac{B\left(z_{I}\right) D\left(z_{K}\right)}{A\left(z_{I}\right) C\left(z_{K}\right)}
$$

only if $z_{I \cup K \cup\{u\}} \in(\partial \mathbb{D})^{|I|+|K|+1}$.

3.3. Proposition. Let $I,\{u\},\{v\}$ be pairwise disjoint and

$$
R\left(z_{I \cup\{u\} \cup\{v\}}\right)=A\left(z_{I}\right)+B\left(z_{I}\right) z_{u}+C\left(z_{I}\right) z_{v}+D\left(z_{I}\right) z_{u} z_{v} .
$$

Define

$$
P\left(z_{I \cup\{u\}}\right)=A\left(z_{I}\right)+D\left(z_{I}\right) z_{u} .
$$

If $R$ satisfies (A), then $P$ satisfies (A).

Let $z_{I \cup\{u\}} \in \mathbb{D}^{|I|+1}$ and $P\left(z_{I \cup\{u\}}\right)=0$. If $R$ satisfies $(\mathrm{A})$, the two roots of the equation

$$
A\left(z_{I}\right)+\left[B\left(z_{I}\right)+C\left(z_{I}\right)\right] z+D\left(z_{I}\right) z^{2}=0
$$

are $\geqq 1$ in absolute value. Therefore

$$
\left|D\left(z_{I}\right) / A\left(z_{I}\right)\right| \leqq 1
$$

and the inequality is strict unless $z_{I} \in(\partial \mathbb{D})^{|I|}$. This is compatible with

$$
1 \leqq\left|z_{u}^{-1}\right|=\left|\frac{D\left(z_{I}\right)}{A\left(z_{I}\right)}\right|
$$

only if $z_{I \cup\{u\}} \in(\partial \mathbb{D})^{|I|+1}$. 
3.4. Proposition. Let $A=I \cup\{u\} ; P$ satisfies (B) if and only if one can write

$$
P\left(z_{A}\right)=A\left(z_{I}\right)+\omega A^{\dagger}\left(z_{I}\right) z_{u} .
$$

Let $a_{X}=c_{X}$ and $b_{X}=c_{X \cup\{u\}}$ for $X \subset I$. (B) is equivalent to $c_{X \cup\{u\}}$ $=\omega c_{A \backslash(X \cup\{u\}}^{*}$, or to $c_{X \cup\{u\}}=\omega c_{I \backslash X}^{*}$ or to $b_{X}=\omega a_{I \backslash X^{\prime}}^{*}$ or to (3.2).

3.5. Corollary. If, in Proposition $3.2, R$ and $S$ satisfy (B) with parameters $\omega_{1}, \omega_{2}$ then $P$ satisfies (B) with parameter $\omega_{1} \omega_{2}$. If in Proposition 3.3, $R$ satisfies (B) with parameter $\omega, P$ satisfies (B) with the same parameter $\omega$.

3.6. Proposition. Let $P$ satisfy $(\mathrm{B})$ and write

$$
P\left(z_{A}\right)=A_{u}\left(z_{\Lambda \backslash\{u\}}\right)+\omega A_{u}^{\dagger}\left(z_{\Lambda \backslash\{u\}}\right) z_{u} .
$$

Then (A) is equivalent to.

(c) For all $u \in A, A_{u}$ does not vanish on $\mathbb{D}^{|A|-1}$.

The implication $(\mathrm{A}) \Rightarrow(\mathrm{c})$ follows from Proposition 3.1. If (c) holds, then $f_{u}=-\omega A_{u}^{\dagger} / A_{u}$ is analytic on $\mathbb{D}^{|A|-1}$ and therefore reaches its maximum on $(\partial \mathbb{D})^{|\Lambda|-1}$. But, for $z_{\Lambda \backslash\{u\}} \in(\partial \mathbb{D})^{|\Lambda|-1}$, (3.1) yields

$$
\left|f_{u}\left(z_{\Lambda \backslash\{u\}}\right)\right|=\left|\frac{\omega z^{\Lambda \backslash\{u\}} A_{u}\left(z_{\Lambda \backslash\{u\}}^{*-1}\right)}{A_{u}\left(z_{A \backslash\{u\}}\right)}\right|=1 .
$$

Therefore if $P\left(z_{A}\right)=0$ and $z_{A} \in \mathbb{D}^{|\Lambda|}$, we have

$$
1 \geqq\left|f_{u}\left(z_{\Lambda \backslash\{u}\right)\right|=\left|z_{u}^{-1}\right| \geqq 1
$$

so that $z_{u} \in \partial \mathbb{D}$, proving (A).

3.7. Corollary. The set of polynomials satisfying (A), (B) is open in the set of polynomials satisfying (B).

If $P$ satisfies (A), (B), there exists $\varepsilon>0$ such that $\left|A_{u}\left(z_{A \mid\langle u\}}\right)\right|>\varepsilon$ for all $u$ and $z_{\Lambda \mid\{u\}} \in \mathbb{D}^{|\Lambda|-1}$ (by compactness of $\mathbb{D}^{|A|-1}$ ). Therefore (c) remains true for polynomials close to $P$.

3.8. Proposition. Consider a spin system on the lattice $\mathbb{Z}^{v}$, and let $\Phi$, $\Psi$ be two translation invariant interactions. The energy of a configuration $\sigma=\left(\sigma_{x}\right)_{x \in A}$ of spins $\sigma_{x}= \pm 1$ in the finite region $\Lambda \subset \mathbb{Z}^{v}$ is given by

$$
U_{\Phi+\lambda \Psi}^{A}(\sigma)=\sum_{\left\{x_{1}, \ldots, x_{k}\right\} \subset A}\left[\Phi_{\left(x_{1}, \ldots, x_{k}\right)}\left(\sigma_{x_{1}}, \ldots, \sigma_{x_{k}}\right)+\lambda \Psi_{\left(x_{1}, \ldots, x_{k}\right)}\left(\sigma_{x_{1}}, \ldots, \sigma_{x_{k}}\right)\right] .
$$

We assume that $\Phi$ and $\Psi$ satisfy the following conditions

(a) The only components of $\Phi$ which do not vanish are $\Phi_{\left(x_{1}, x_{2}\right)}\left(\sigma_{x_{1}}, \sigma_{x_{2}}\right)$ $=\varphi\left(x_{2}-x_{1}\right) \times\left(1-\sigma_{x_{1}} \sigma_{x_{2}}\right)$. Also, $\varphi \geqq 0$, i.e., $\Phi$ is a ferromagnetic pair interaction. Furthermore $\sum_{x \neq 0} \varphi(x)<+\infty$, and the lattice $\mathbb{Z}^{v}$ is connected by the bonds $x_{1}-x_{2}$ such that $\varphi\left(x_{2}-x_{1}\right)>0$ (this means that for any $x, y \in \mathbb{Z}^{v}$ there is a chain $x=x_{0}, x_{1}, \ldots, x_{n}=y$ such that $\varphi\left(x_{m}-x_{m-1}\right)>0$ for $m=1, \ldots, n)$. 
(b) $\Psi$ has finite range (i.e. there is a finite set $\Delta \subset \mathbb{Z}^{v}$ such that $\Psi_{\left(x_{1}, \ldots, x_{k}\right)}\left(\sigma_{x_{1}}, \ldots, \sigma_{x_{k}}\right)=0$ unless $x_{i}-x_{1} \in \Delta$ for $i=2, \ldots, k$ and

$$
\begin{gathered}
\Psi_{\left(x_{1}, \ldots, x_{k}\right)}(1, \ldots, 1)=0, \\
\Psi_{\left(x_{1}, \ldots, x_{k}\right)}\left(\sigma_{x_{1}}, \ldots, \sigma_{x_{k}}\right)=\Psi_{\left(x_{1}, \ldots, x_{k}\right)}\left(-\sigma_{x_{1}}, \ldots,-\sigma_{x_{k}}\right)
\end{gathered}
$$

(the last property expresses that the interaction $\Psi$ is even).

Under these conditions, one can construct $V$ differing from $U_{\Phi+\lambda \Psi}$ by a boundary term (without effect in the thermodynamic limit) and choose $\varepsilon>0$ (for given $\beta>0$ ) so that the zeroes of the partition function

$$
Z_{\Lambda}(z)=\sum_{\sigma} z^{\frac{1}{2}\left(\sum_{x \in A} \sigma_{x}+1\right)} e^{-\beta V(\sigma)}
$$

have absolute value 1 when $|\lambda|<\varepsilon$.

We want to prove that $Z_{A}$ can be obtained by contraction of a product of polynomials $P_{i}$ satisfying conditions (A) and (B). Given $x \in \mathbb{Z}^{v}$ let

$$
B_{\Psi, x}(\sigma)=\sum_{k} \sum_{\left\{x, x_{2}, \ldots, x_{k}\right\} \subset x+\Delta} \frac{1}{k} \Psi_{\left(x, x_{2}, \ldots, x_{k}\right)}\left(\sigma_{x}, \ldots, \sigma_{x_{k}}\right) .
$$

Then, apart from boundary terms, $U_{\Psi}^{A}(\sigma)$ is equal to

$$
\sum_{x \in \Lambda} B_{\Psi, x}(\sigma) \text {. }
$$

Let now $\Gamma$ be a finite subset of $\mathbb{Z}^{v}$ containing $\Delta$ and which is connected by bonds $x_{1}-x_{2}$ such that $\varphi\left(x_{2}-x_{1}\right)>0$. If $N$ is the number of points of $\Gamma$, let

$$
A_{\Phi, x}(\sigma)=\frac{1}{N} \sum_{\left\{x_{1}, x_{2}\right\} \subset x+\Gamma} \varphi\left(x_{2}-x_{1}\right) \times\left(1-\sigma_{x_{1}} \sigma_{x_{2}}\right)
$$

then, apart from boundary terms $U_{\Phi}^{A}(\sigma)$ is equal to

$$
\sum_{x \in A} A_{\Phi, x}(\sigma)+W(\sigma)
$$

where the energy function $W$ is derived from a ferromagnetic pair interaction. Up to boundary terms, $U_{\Phi+\lambda \Psi}^{A}$ is thus equal to

$$
V=\sum_{x \in \Lambda}\left(A_{\Phi, x}+\lambda B_{\Psi, x}\right)+W .
$$

The partition function $P_{x}$ for the region $x+\Gamma$, constructed with the energy $A_{\Phi, x}+\lambda B_{\Psi, x}$ satisfies (B) with $\omega=1$. So does the partition function $P^{\prime}$ constructed with the energy $W$. By Proposition 3.2 and Proposition 3.3, when $\lambda=0, P_{x}$ satisfies (A). By Corollary 3.7 there is thus $\varepsilon>0$ such that $P_{x}$ satisfies $(\mathrm{A})$ when $|\lambda|<\varepsilon$. On the other hand $P^{\prime}$ is a limit of partition functions satisfying (A). Since $Z_{A}$ is obtained by contraction from the product of $P^{\prime}$ and the $P_{x}$, it is (by 3.2 and 3.3) a limit of partition functions satisfying (A), and therefore $Z_{A}(z)=0$ implies $|z|=1$. 


\section{References}

1. Abe, Y., Katsura, S.: Distribution of zeroes of the partition function in the complex temperature plane II. Progr. Theoret. Phys. 43, 1402-1403 (1970).

2. Asano, T.: The rigorous theorems for the Heisenberg ferromagnets. J. Phys. Soc. Japan 29, 350--359 (1970).

3. Borchers, H.J.: Über die Vollständigkeit Lorentzinvarianter Felder in einer zeitartigen Röhre. Nuovo Cim. 19, 787--796 (1961).

4. DiLiberto',F.: On the uniqueness of the equilibrium state for antiferromagnetic Ising spin system in the phase transition region. Preprint.

5. Dobrushin, R.L.: The problem of uniqueness of a Gibbsian random field and the problem of phase transitions. Funkts. Anal. i Prilozh. 2(4), 44-57 (1968).

6. Epstein, H.: Thèse. A paraître.

7. Fisher,M.E.: The nature of critical points, pp. 1-159 in Lectures in Theoretical Physics 7c. Boulder: University of Colorado Press 1964.

8. Ginibre, J.: On some recent work of Dobrushin. In: Systèmes à un nombre infini de degrés de liberté, pp. 163-175. Colloque CNRS No. 181 (CNRS, Paris, 1970).

9. Glaser, V., Bros, J.: L'enveloppe d'holomorphie de l'union de deux polycercles. Preprint, CERN 1961.

10. Heilmann, O.J.: Location of the zeroes of the grand partition function of certain classes of lattice gases. Stud. in Appl. Math. 50, 385 (1971).

11. Heilmann, O.J., Lieb,E.H.: Theory of monomerdimer systems. Commun. math. Phys. 25, 190-232 (1972).

12. Lee, T.D., Yang, C.N.: Statistical theory of equations of state and phase translations. II. Lattice gas and Ising model. Phys. Rev. 87, $410-419$ (1952).

13. Lieb,E.H., Ruelle, D.: A property of zeroes of the partition function for Ising spin systems. J. Math. Phys. 13, $781-784$ (1972).

14. Miracle-Sole, S.: Private communication.

15. Penrose, O., Elvey,J.S.N.: The Yang-Lee distribution of zeros for a classical onedimensional fluid. J. Phys. A. 1, $661-674$ (1968).

16. Polya, G., Szegö, G.: Aufgaben und Lehrsätze aus der Analysis, 2 Vol., 3 rd ed. BerlinGöttingen-Heidelberg: Springer 1964.

17. Ruelle, D.: Extension of the Lee-Yang circle theorem. Phys. Rev. Letters 26, 303-304 (1971).

18. Runnels, L.K., Hubbard, J.B.: Applications of the Yang-Lee-Ruelle theory to hardcore lattice gases. J. Stat. Phys. 6, 1--20 (1972).

19. Suzuki,M., Fisher, M.E.: Zeros of the partition function for the Heisenberg and general Ising models. J. Math. Phys. 12, 235-246 (1971).

20. Yang, C.N., Lee, T.D.: Statistical theory of equations of state and phase transitions. I. Theory of condensation. Phys. Rev. 87, 404-409 (1952).

D. Ruelle

Institut des Hautes Etudes Scientifiques

F-91440 Bures-sur-Yvette, France 
